\title{
High-Resolution Transmission Electron Microscopy with Bright Microsecond Electron Pulses
}

Pavel Olshin, Gabriele Bongiovanni, Marcel Drabbels and Ulrich Lorenz

LND, EPFL, Switzerland, United States

Transmission electron microscopy at atomic-resolution is a crucial tool in a variety of fields, ranging from materials science to biology. [1,2] Fast electron cameras have recently enabled high-speed observations atomic resolution. [3] Moreover, they have made it possible to correct for sample drift by recording high-resolution micrographs as movies. Even higher time resolution, can be achieved by imaging with short electron pulses. [4-6] Yet, the potential of electron pulses for atomic-resolution imaging has largely remained unexplored. This is in part due to the difficulty of generating pulses that are both intense enough to the capture the image information in a short amount of time, but also coherent enough, to allow for atomic-resolution imaging. Here, we demonstrate the generation of intense, high-brightness electron pulses of microsecond duration from a Schottky field emitter whose emission current we temporarily drive to near its limit. [7] We show that such pulses enable drift-corrected imaging at atomic resolution in the presence of large amounts of drift. [8] Furthermore, they allow us to capture atomic-resolution snapshots of fast, irreverible processes, which we use to elucidate the mechanism of the high-temperature amorphization of perovskite nanocrystals. [8] As illustrated in Fig. 1, we generate electron pulses by illuminating the Schottky emitter of our microscope with a microsecond laser pulse $(532 \mathrm{~nm})$, which briefly heats the emitter tip to an estimated temperature of $2500 \mathrm{~K}$ (Ref. 7), well beyond the typical safe operating temperature. This temporarily boosts the emission current to near its limit (almost four times) and increases the brightness of the electron beam by a factor of 1.7, without damaging to the emitter. The laser-boosted electron beam is then chopped with an electrostatic deflector to obtain pulses of microsecond duration, and as short as $100 \mathrm{~ns}$.

We show that such intense, high-brightness electron pulses enable drift-corrected imaging at atomic resolution in the presence of otherwise prohibitively large amounts of drift, as they are for example frequently encountered in in situelectron microscopy experiments. [9] In order to induce a reproducible drift, we irradiate the sample with a laser pulse in situ, as shown in Fig. 1b. Under such conditions, which create a drift of $9 \mathrm{~nm} / \mathrm{s}$, drift-corrected images of gold nanoparticles recorded with a continuous electron beam $(25 \mathrm{~Hz}$ camera frame rate) appear blurry (Fig. 2a). However, if we instead deliver the same electron dose per camera frame in an intense $480 \mu$ s pulse (Fig. 2xx), lattice fringes become clearly visible.

We also demonstrate the potential of our electron pulses to capture atomic-resolution snapshots of irreversible transformations of nanoparticles on the microsecond timescale. As an example, we study the mechanism of the high-tempearture amorphization of perovskite nanocrystals, a phenomenon that is associated with the degradation of their optoelectronic properties in high-power devices. [10] Under in situlaser irradiation, the perovskite nanoparticle in Fig. 2c turns amorphous (Fig. 2e). A snapshot acquired during the laser pulse (Fig. 2d) reveals details of the mechanism, showing that crystallinity is lost at the boundaries first, before the amorphization process propagates towards the bulk of the crystal. [8]

P.O. and G.B. contributed equally. This work was supported by the ERC Starting Grant 759145 and by the Swiss National Foundation Grants PP00P2_163681 and 206021_183295. 
a

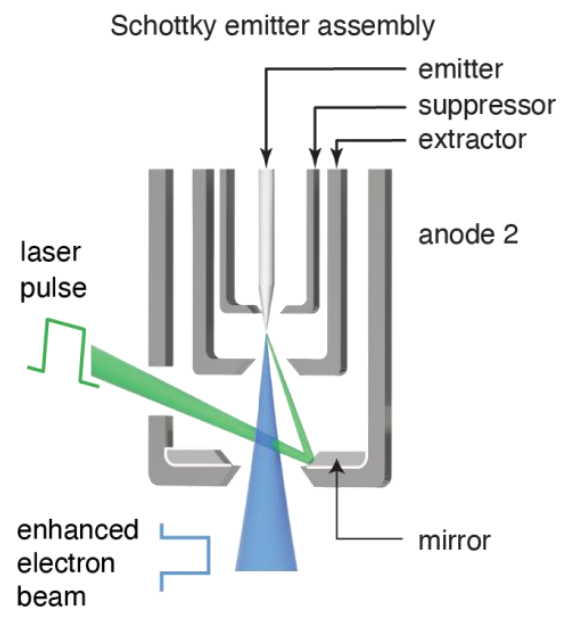

b

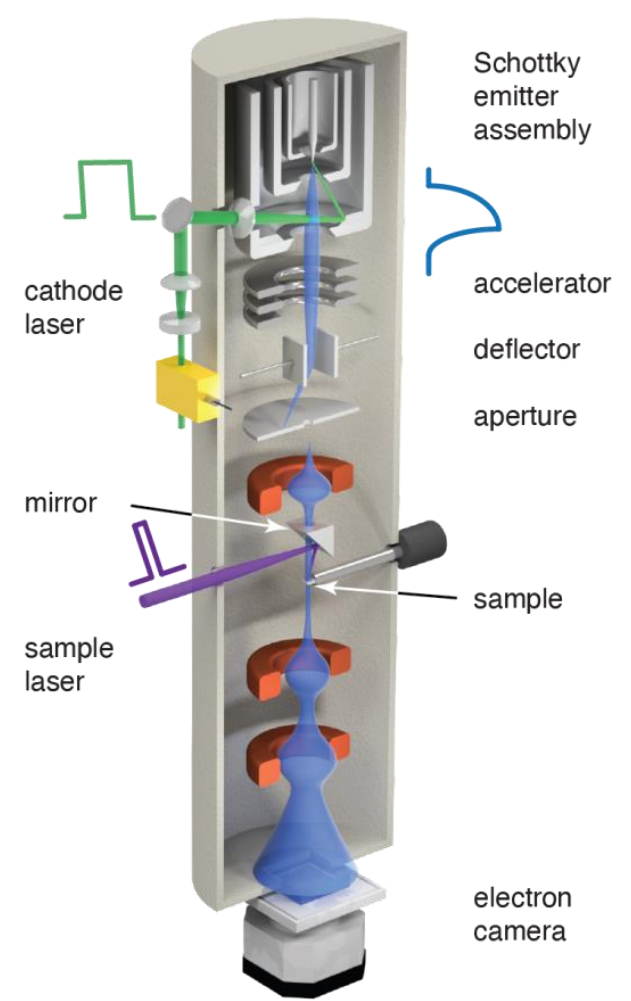

Figure 1. Figure 1. Generationof intense microsecond electron pulses of high brightness. (a), The temperature of the Schottky emitter is raised thanks to a microsecond laser pulse. (b), Illustration of the modified electron microscope. The intense electron pulse in (a) is chopped using an electrostatic deflector. Dynamics at the sample are initiated with a second laser pulse. 


\section{Drift-corrected imaging with microsecond electron pulses Drift velocity: $9 \mathrm{~nm} / \mathrm{s}$}
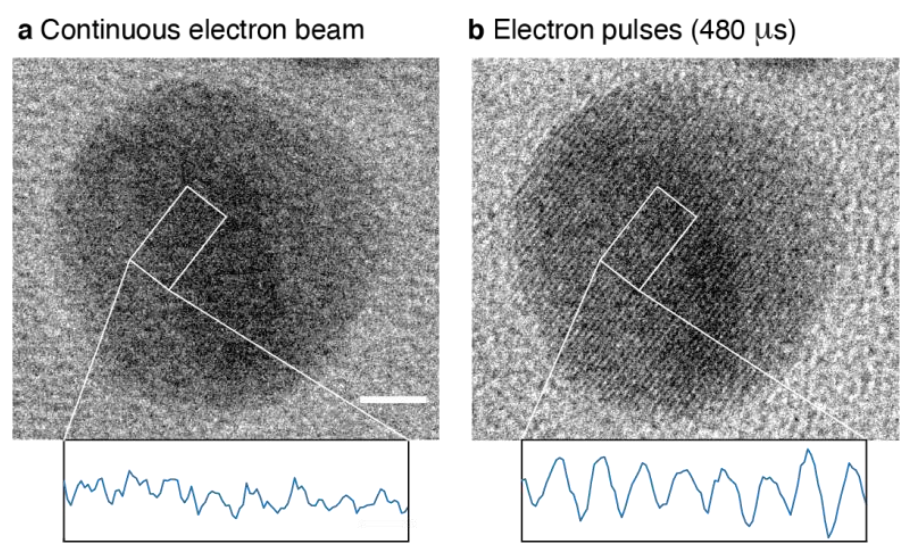

Amorphization of a perovskite nanocrystal

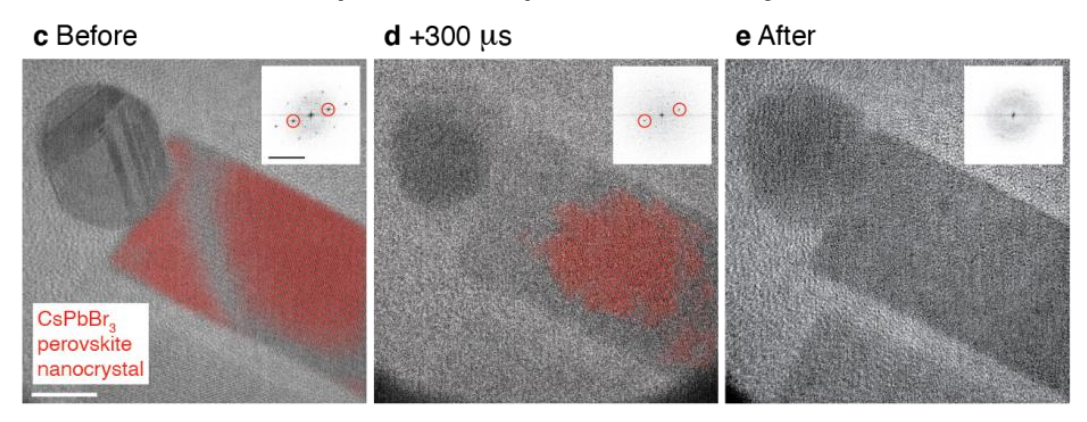

Figure 2. Figure 2. Examples of applications of bright microsecond electron pulses. (a,b) Drift-corrected micrograph of a gold nanoparticle acquired in the presence of high drift with the continuous electron beam (a) and with a single $480 \mu$ s pulses per frame (b). The insets show how lattice fringes can only be seen when the beam is pulsed. Scale bar, $3 \mathrm{~nm}$. (c-e), High-temperature amorphization of a perovskite nanocrystal. The nanocrystal in (c) is rendered amorphous with a short laser pulse $(500 \mu \mathrm{s})$, during which a snapshot is taken (d). The single-shot reveals that the amorphization takes place first at the edges of the crystal, while the center remains crystalline. The nanocrystal is completely amorphous at the end of the laser pulse (e). Scale bars, 10 $\mathrm{nm}$ and $5 \mathrm{~nm}-1$.

\section{References}

(1) Urban, K. W. Is Science Prepared for Atomic-Resolution Electron Microscopy? Nat. Mater.2009, $8(4), 260-262$.

(2) Nakane, T., Kotecha, A., Sente, A. et al. Single-particle cryo-EM at atomic resolution. Nature 587, 152-156 (2020).

(3) Cheng, Y.; Grigorieff, N.; Penczek, P. A.; Walz, T. A Primer to Single-Particle Cryo-Electron Microscopy. Cell2015, 161(3), 438-449.

(4) Cremons, D. R.; Plemmons, D. A.; Flannigan, D. J. Femtosecond Electron Imaging of DefectModulated Phonon Dynamics. Nat. Commun.2016, 7.

(5) Feist, A.; Bach, N.; Rubiano da Silva, N.; Danz, T.; Möller, M.; Priebe, K. E.; Domröse, T.; Gatzmann, J. G.; Rost, S.; Schauss, J.; Strauch, S.; Bormann, R.; Sivis, M.; Schäfer, S.; Ropers, C. Ultrafast Transmission Electron Microscopy Using a Laser-Driven Field Emitter: Femtosecond Resolution with a High Coherence Electron Beam. Ultramicroscopy 2017, 176, 63-73. 
(6) Picher, M.; Bücker, K.; LaGrange, T.; Banhart, F. Imaging and Electron Energy-Loss Spectroscopy Using Single Nanosecond Electron Pulses. Ultramicroscopy 2018, 188, 41-47.

(7) Bongiovanni, G.; Olshin, P. K.; Drabbels, M.; Lorenz, U. J. Intense Microsecond Electron Pulses from a Schottky Emitter. Appl. Phys. Lett.2020, 116(23), 234103.

(8) Olshin, P. K.; Bongiovanni, G.; Drabbels, M.; Lorenz, U. J. Atomic-Resolution Imaging of Fast Nanoscale Dynamics with Bright Microsecond Electron Pulses. Nano Lett. 2021, 21(1), 612-618.

(9) Taheri, M. L.; Stach, E. A.; Arslan, I.; Crozier, P. A.; Kabius, B. C.; LaGrange, T.; Minor, A. M.; Takeda, S.; Tanase, M.; Wagner, J. B.; Sharma, R. Current Status and Future Directions for in Situ Transmission Electron Microscopy. Ultramicroscopy 2016, 170, 86-95.

(10) Akkerman, Q. A.; Rainò, G.; Kovalenko, M. V.; Manna, L. Genesis, Challenges and Opportunities for Colloidal Lead Halide Perovskite Nanocrystals. Nat. Mater. 2018, 17(5), 394-405. 